\title{
FOURIER TRANSFORMS OF $B$-SPLINES AND FUNDAMENTAL SPLINES FOR CARDINAL HERMITE INTERPOLATIONS
}

\author{
S. L. LEE
}

\begin{abstract}
Using the exponential Hermite Euler splines we compute the Fourier transforms of the $B$-splines and fundamental splines for Cardinal Hermite Interpolation, introduced by Schoenberg and Sharma and Lipow and Schoenberg respectively.
\end{abstract}

Introduction. Let $n, r$ be positive integers such that $n>2 r-1$. The class $\S_{n, r}$ of cardinal splines of degree $n$ with integer knots of multiplicity $r$ consists of functions $S(x)$ which are polynomials of degree $n$ in each of the intervals $[\nu, \nu+1](\nu=0, \pm 1, \pm 2, \cdots)$ and belong to class $C^{n-r}(-\infty, \infty)$. The Cardinal Hermite Interpolation Problem (C.H.I.P.) was first considered by P. Lipow and I. J. Schoenberg [1] whose main result states that for any set of $r$ bi-infinite sequences $\left(y_{v}^{(s)}\right)(s=0,1, \ldots, r-1)$ such that

$$
y_{\nu}^{(s)}=O\left(|\nu|^{\gamma}\right) \quad(s=0,1, \ldots, r-1) \text { for some } \gamma>0,
$$

there is a unique $S_{2 m-1}(x) \in \widehat{S}_{2 m-1, r}$ such that

$$
S_{2 m-1}^{(s)}(\nu)=y_{\nu}^{(s)} \quad(s=0,1, \ldots, r-1) \text { for all integers } \nu .
$$

Furthermore, the spline $S_{2 m-1}(x)$ is given by the Hermite interpolation formula

$$
S_{2 m-1}(x)=\sum_{s=0}^{r-1} \sum_{\nu=-\infty}^{\infty} y_{\nu}^{(s)} L_{2 m-1, r, s}(x-\nu),
$$

where the fundamental splines $L_{2 m-1, r, s}(x)(s=0,1, \ldots, r-1)$ are uniquely determined by the conditions

$$
\left\{\begin{array}{l}
L_{2 m-1, r, s}^{(\rho)}(\nu)=0 \quad(\rho=0,1, \ldots, r-1) \quad \forall \text { integers } \nu \neq 0 \\
L_{2 m-1, r, s}^{(\rho)}(0)=\delta(\rho, s), \quad \text { Kronecker delta. }
\end{array}\right.
$$

Subsequently, Schoenberg and Sharma [11] introduced the $B$-splines $N_{s}(x)$ $(s=0,1, \ldots, r-1)$ which belong to the space $\mathcal{S}_{2 m-1, r}^{(s)}=\left\{S(x) \in \mathcal{S}_{2 m-1, r}\right.$ : $S^{(\rho)}(\nu)=0(\rho=0,1, \ldots, r-1, \rho \neq s) \forall$ integers $\left.\nu\right\}$, have support in $(-(m-r+1),(m-r+1))$ and satisfy the interpolatory properties

$$
N_{s}^{(s)}(\nu)= \begin{cases}C_{\nu}(\nu=-(m-r), \ldots,(m-r)) \\ 0 \quad \text { otherwise, }\end{cases}
$$

where $C_{\nu}$ are the coefficients of the monic reciprocal polynomials

Received by the editors March 3, 1975.

AMS (MOS) subject classifications (1970). Primary 41A05, 41A15; Secondary 42A68.

Key words and phrases. Fourier transforms, spline functions, Cardinal Hermite Interpolation.

( ) American Mathematical Society 1976 


$$
\Pi_{2 m-1, r}(\lambda)=\sum_{\nu=0}^{2 m-2 r} C_{\nu-(m-r)} \lambda^{\nu} \quad\left(C_{0}>0\right) .
$$

One of the most important properties of the $B$-splines (see [2], [11]) asserts that every $S(x) \in \varsigma_{2 m-1, r}^{(s)}$ admits a unique representation of the form

$$
S(x)=\sum_{\nu=-\infty}^{\infty} a_{\nu} N_{s}(x-\nu) \text {. }
$$

For the case $r=1$, the corresponding interpolation problem, called the Cardinal Interpolation Problem (C.I.P.) dates back to the first paper on spline functions of I. J. Schoenberg [6] and has been extensively studied by him in several recent works (see [7], [8], [10]). The theory of C.I.P. (the case $r=1$ ) is very much enriched by the help of the Fourier transforms of the $B$-splines and fundamental splines (see [5], [6], [7], [12], [13]) viz.

$$
\begin{aligned}
N_{0}(x) & =\frac{(2 m-1) !}{2 \pi} \int_{-\infty}^{\infty} e^{i u x}\left\{\frac{2 \sin u / 2}{u}\right\}^{2 m} d u, \\
L_{2 m-1,1,0}(x) & =\frac{1}{2 \pi} \int_{-\infty}^{\infty} e^{i u x} \frac{u^{-2 m}}{\alpha_{2 m}(u)} d u,
\end{aligned}
$$

where

$$
\alpha_{n}(u)=\sum_{k=-\infty}^{\infty} \frac{1}{(u+2 \pi k)^{n}}
$$

The purpose of this paper is to compute Fourier transforms of the fundamental splines $L_{2 m-1, r, s}(x)$ and $B$-splines $N_{s}(x)$ for the general C.H.I.P. This is achieved with the help of the exponential Hermite Euler splines introduced in [3]. More precisely, we shall prove the following theorems.

THEOREM 1. The Fourier integral representations of the fundamental splines are given by

$$
\begin{aligned}
L_{2 m-1, r, s}(x)=\frac{(-i)^{s}}{2 \pi} \int_{-\infty}^{\infty} e^{i u x} \frac{H_{r, s}\left(\alpha_{2 m}(u)\right)}{H_{r}\left(\alpha_{2 m}(u)\right)} d u & \\
& (s=0,1, \ldots, r-1)
\end{aligned}
$$

where $H_{r}\left(a_{n}\right)$ denotes the Hankel determinant given by

$$
H_{r}\left(a_{n}\right)=\left|\begin{array}{llll}
a_{n} & a_{n-1} & \cdots & a_{n-r+1} \\
a_{n-1} & a_{n-2} & \cdots & a_{n-r} \\
\cdot & \cdot & & \cdot \\
\cdot & \cdot & & \cdot \\
\cdot & \cdot & & \cdot \\
a_{n-r+1} & a_{n-r} & \cdots & a_{n-2 r+2}
\end{array}\right|
$$

and $H_{r, s}\left(\alpha_{2 m}(u)\right)$ is obtained from $H_{r}\left(\alpha_{2 m}(u)\right)$ by replacing the $(s+1)$ th column $(s=0,1, \ldots, r-1)$ by the column vector

$$
\left(1 / u^{2 m}, 1 / u^{2 m-1}, \ldots, 1 / u^{2 m-r+1}\right)^{T} \text {. }
$$

THEOREM 2. The Fourier integral representations of the B-splines are given by 


$$
N_{s}(x)=(-i)^{s} \frac{K(m, r)}{2 \pi} \int_{-\infty}^{\infty} e^{i u x}\left(2 \sin \frac{u}{2}\right)^{2 m} H_{r, s}\left(\alpha_{2 m}(u)\right) d u
$$

where

$$
K(m, r)=(-1)^{m(r+1)} \frac{(2 m-1) !(2 m-2) ! \cdots(2 m-r) !}{1 ! 2 ! \cdots(r-1) !}
$$

In $\S 1$ we give the preliminaries required to prove Theorems 1 and 2, while in the last section we mention a formula to approximate the Fourier transform of a given function.

1. Preliminaries. The tool for our computations of the Fourier transforms (11) and (13) are the exponential Hermite Euler splines $S_{n, r, s}(x ; \lambda)(s=0$, $1, \ldots, r-1)$ defined by

$$
\left\{\begin{array}{l}
S_{n, r, s}(x ; \lambda)=A_{n, r, s}(x ; \lambda) / H_{r}\left(A_{n}(0 ; \lambda) / n !\right) \quad(0 \leqslant x \leqslant 1) \quad \text { and } \\
S_{n, r, s}(x+1 ; \lambda)=\lambda S_{n, r, s}(x ; \lambda) \quad \forall \text { real } x,
\end{array}\right.
$$

where

$$
\begin{aligned}
& A_{n, r, s}(x ; \lambda) \\
& =\left[\begin{array}{ccccccc}
\frac{A_{n}(0 ; \lambda)}{n !} & \cdots & \frac{A_{n-s+1}(0 ; \lambda)}{(n-s+1) !} & \frac{A_{n}(x ; \lambda)}{n !} & \frac{A_{n-s-1}(0 ; \lambda)}{(n-s-1) !} & \cdots & \frac{A_{n-r+1}(0 ; \lambda)}{(n-r+1) !} \\
\frac{A_{n-1}(0 ; \lambda)}{(n-1) !} & \cdots & \frac{A_{n-s}(0 ; \lambda)}{(n-s) !} & \frac{A_{n-1}(x ; \lambda)}{(n-1) !} & \frac{A_{n-s-2}(0 ; \lambda)}{(n-s-2) !} & \cdots & \frac{A_{n-r}(0 ; \lambda)}{(n-r) !} \\
\vdots & & \vdots & \vdots & \vdots & & \vdots \\
\frac{A_{n-r+1}(0 ; \lambda)}{(n-r+1) !} & \cdots & \frac{A_{n-r-s+2}(0 ; \lambda)}{(n-r-s+2) !} & \frac{A_{n-r+1}(x ; \lambda)}{(n-r+1) !} & \frac{A_{n-r-s}(0 ; \lambda)}{(n-r-s) !} & \cdots & \frac{A_{n-2 r+2}(0 ; \lambda)}{(n-2 r+2) !}
\end{array}\right]
\end{aligned}
$$

and $A_{n}(x ; \lambda)$ are the exponential Euler polynomials (see [9]). Furthermore $S_{n, r, s}(x ; \lambda)$ satisfy the following interpolatory conditions (see [3]):

$$
\begin{cases}S_{n, r, s}^{(\rho)}(\nu ; \lambda)=0 & (\rho=0,1, \ldots, r-1, \rho \neq s) \text { and } \\ S_{n, r, s}^{(s)}(\nu ; \lambda)=\lambda^{\nu} & (\nu=0, \pm 1, \pm 2, \ldots) .\end{cases}
$$

We shall also need the following relation given in [4]:

$$
H_{r}\left(A_{n}(0 ; \lambda) / n !\right)=(-1)^{[r / 2]+(r-1)(n+1)} \frac{C(n, r) \Pi_{n, r}(\lambda)}{(\lambda-1)^{n-r+1}},
$$

where $C(n, r)=1 ! 2 ! \cdots(r-1) ! / n !(n-1) ! \cdots(n-r+1) !$, and $\Pi_{n, r}(\lambda)$ are given by $(6)$.

2. Proof of Theorem 1. Consider the bounded exponential Hermite Euler splines $S_{2 m-1, r, s}\left(x ; e^{i u}\right)$ for $0<u<2 \pi$ if $r$ is even and $-\pi<u<\pi$ if $r$ is odd. Following Schoenberg it is easy to show that the functions

$$
\begin{cases}\frac{1}{2 \pi} \int_{0}^{2 \pi} S_{2 m-1, r, s}\left(x ; e^{i u}\right) d u & (r \text { even }) \\ \frac{1}{2 \pi} \int_{-\pi}^{\pi} S_{2 m-1, r, s}\left(x ; e^{i u}\right) d u & (r \text { odd })\end{cases}
$$


are spline functions belonging to $\varsigma_{2 m-1, r}$. Furthermore, these functions satisfy (4) in view of the interpolating conditions $S_{2 m-1, r, s}^{(s)}\left(\nu ; e^{i u}\right)=e^{i v u}$ and $S_{2 m-1, r, s}^{(\rho)}\left(\nu ; e^{i u}\right)=0(\rho=0,1, \ldots, r-1, \rho \neq s)$ for all integers $\nu$. Hence, for $s=0,1, \ldots, r-1$,

$$
L_{2 m-1, r, s}(x)= \begin{cases}\frac{1}{2 \pi} \int_{0}^{2 \pi} S_{2 m-1, r, s}\left(x ; e^{i u}\right) d u & (r \text { even }) \\ \frac{1}{2 \pi} \int_{-\pi}^{\pi} S_{2 m-1, r, s}\left(x ; e^{i u}\right) d u & (r \text { odd })\end{cases}
$$

Using the expansion

$$
\frac{A_{n}\left(x ; e^{i u}\right)}{n !}=\left(e^{i u}-1\right) e^{-i u} e^{i u x} \sum_{k=-\infty}^{\infty} \frac{e^{2 \pi i k x}}{(u i+2 \pi k i)^{n+1}}
$$

$$
(0 \leqslant x \leqslant 1)
$$

(see [9]), it follows from (1.1) that

$$
S_{2 m-1, r, s}\left(x ; e^{i u}\right) e^{-i u x}=(-i)^{s} \sum_{k=-\infty}^{\infty} e^{2 \pi k i x} \frac{\Delta_{2 m, k, s}(x ; u)}{H_{r}\left(\alpha_{2 m}(u)\right)},
$$

where $\Delta_{2 m, k, s}(x ; u)$ is obtained from $H_{r}\left(\alpha_{2 m}(u)\right)$ by replacing the $s$ th column by the column vector

$$
\left(\frac{1}{(u+2 \pi k)^{2 m}}, \frac{1}{(u+2 \pi k)^{2 m-1}}, \ldots, \frac{1}{(u+2 \pi k)^{2 m-r+1}}\right)^{T} .
$$

Substituting (2.3) into (2.2) and performing a change of variable after interchanging the order of integration and summation we obtain (11).

REMARK. When $r=1$ (in which case $s$ can take only the value zero), $H_{1,0}\left(\alpha_{2 m}(u)\right)=u^{-2 m}$ and so (11) reduces to (9).

3. Proof of Theorem 2. From (3) and (5) we can write

$$
N_{s}(x)=\sum_{\nu=-(m-r)}^{(m-r)} C_{\nu} L_{2 m-1, r, s}(x-\nu),
$$

and using (11), after some simplifications, we obtain

$$
N_{s}(x)=\frac{(-i)^{s}}{2 \pi} \int_{-\infty}^{\infty} e^{-(m-r) i u} \Pi_{2 m-1, r}\left(e^{i u}\right) e^{i u x} \frac{H_{r, s}\left(\alpha_{2 m}(u)\right)}{H_{r}\left(\alpha_{2 m}(u)\right)} d u
$$

Using (1.3), we can write

$$
\Pi_{2 m-1, r}\left(e^{i u}\right)=(-1)^{[r / 2]+m(r+1)} K(m, r)\left(e^{i u}-1\right)^{2 m-r} H_{r}\left(A_{n}\left(0 ; e^{i u}\right) / n !\right) .
$$

Then using expansion (2.3) we obtain

$$
\Pi_{2 m-1, r}\left(e^{i u}\right)=(-i)^{2 m r} K(m, r) \frac{\left(e^{i u}-1\right)^{2 m}}{e^{i u r}} H_{r}\left(\alpha_{2 m}(u)\right),
$$

where $K(m, r)$ is given by (14). Substituting (3.2) into (3.1), we obtain (13).

REMARK. When $r=1$ (in which case $s=0$ ), (13) reduces to (8). 
4. An approximation of Fourier transform. Let $f(x)$ be $(2 m-r+1)$ times continuously differentiable and $f^{(s)}(x) \in L_{1}(\mathbf{R})(s=0,1, \ldots, r-1)$. Using the same method as Silliman [13] it is possible to obtain a formula to approximate the Fourier transform

$$
F(u)=\int_{-\infty}^{\infty} e^{i u x} f(x) d x .
$$

Indeed, let $S_{2 m-1}(x)$ be the unique solution of the C.H.I.P. to the data $\left\{f^{(s)}(\nu)\right\}(s=0,1, \ldots, r-1)$. Then

$$
S_{2 m-1}(x)=\sum_{\nu=-\infty}^{\infty} \sum_{s=0}^{r-1} f^{(s)}(\nu) L_{2 m-1, r, s}(x-\nu) .
$$

Using Theorem 1, the Fourier transform of $S_{2 m-1}(x)$ is given by

$$
\int_{-\infty}^{\infty} S_{2 m-1}(x) e^{i u x} d x=\sum_{s=0}^{r-1}(-1)^{s} \frac{H_{r, s}\left(\alpha_{2 m}(u)\right)}{H_{r}\left(\alpha_{2 m}(u)\right)} \sum_{\nu=-\infty}^{\infty} f^{(s)}(\nu) e^{i \nu u},
$$

which serves as an approximation to $F(u)$. We can write

$$
\int_{-\infty}^{\infty} e^{i u x} f(x) d x=\sum_{s=0}^{r-1}(-1)^{s} \frac{H_{r, s}\left(\alpha_{2 m}(u)\right)}{H_{r}\left(\alpha_{2 m}(u)\right)} \sum_{\nu=-\infty}^{\infty} f^{(s)}(\nu) e^{i \nu u}+R_{f}(u)
$$

were $R_{f}(u)$ is the error term.

ACKNOWLEDGMENT. A special case $(r=2)$ of the results of this paper forms part of the author's dissertation written under the supervision of Professor A. Sharma at the University of Alberta, Edmonton. The author wishes to thank Professor A. Sharma for his help and guidance during the writing of the thesis.

\section{REFERENCES}

1. P. Lipow and I. J. Schoenberg, Cardinal interpolation and spline functions. III. Cardinal Hermite interpolation, J. Linear Algebra and Appl. 6(1973), 273-304.

2. S. L. Lee, B-splines for cardinal Hermite interpolation, J. Linear Algebra and Appl. (to appear).

3. Exponential Hermite Euler splines, J. Approximation Theory (to appear).

4. S. L. Lee and A. Sharma, Cardinal lacunary interpolation by g-splines. I. The characteristic polynomials, J. Approximation Theory (to appear).

5. M. J. Marsden, F. Richards and S. Riemenschneider, Cardinal spline interpolation operators on $l^{p}$ data, Indiana Math. J. 24 (1975), 677-689.

6. I. J. Schoenberg, Contributions to the problem of approximation of equidistant data by analytic functions. Part A. On the problem of smoothing or graduation. A first class of analytic approximation formulae; Part B. On the problem of osculatory interpolation. A second class of analytic approximation formulae, Quart. Appl. Math. 4(1946), 45-99, 112-141. MR 7, 487; 8, 55.

7. Cardinal interpolation and spline functions, J. Approximation Theory 2(1969), 167-206. MR 41 \#2266.

8. Cardinal interpolation and spline functions. II, J. Approximation Theory 6(1972), $404-420$.

9. , Cardinal interpolation and spline functions. IV. The exponential Euler splines, Proc. Oberwolfach Conf., 1971, ISNM 20(1972), 382-402.

10. , Cardinal spline interpolation, Regional Conf. Ser. in Appl. Math., no. 12, SIAM, Philadelphia, Pa., 1973. 
11. I. J. Schoenberg and A. Sharma, Cardinal interpolation and spline functions. V. B-splines for cardinal Hermite interpolation, J. Linear Algebra and Appl. 7(1973), 1-42.

12. F. Richards, The Lebesgue constants for cardinal spline interpolation, MRC Report \#1364, University of Wisconsin, 1973.

13. S. D. Silliman, The numerical evaluation by splines of the Fourier transform and the Laplace transform, MRC Report \#1183, University of Wisconsin, 1972.

School of Mathematical Sciences, Science University of Malaysia, Penang, Malaysia 